\title{
Calculating the polarization lifetime from the Thomas-Bargmann-Michel-Telegdi equation
}

\author{
V. H. Ranjbar®* \\ Brookhaven National Lab, Upton, New York 11973, USA
}

(Received 10 April 2019; published 26 September 2019)

\begin{abstract}
It was observed at the Relativistic Heavy Ion Collider that the polarization of the stored beam decays by 0.17 to $3 \%$ per hour. This effect could not be simulated up to now since the simulation time for an exact calculation exceeded the computer capacity. To solve this problem the action of the whole arc was considered as one element acting on the spin and not, as before, each optical element in the arc individually (called lattice independent integration). In addition to former simulations the coupling of synchrotron and betatron motions in the beam interaction regions and their action on the polarization were taken into account (called addition of longitudinal dynamics). It is shown that with these two changes the action on the polarization life time can be explained.
\end{abstract}

DOI: 10.1103/PhysRevAccelBeams.22.091001

\section{INTRODUCTION}

The Relativistic Heavy Ion Collider (RHIC) is designed to study various aspects of quark and gluon matter. One of the important programs involves colliding intense beams of polarized protons. Collisions with highly polarized protons is necessary to study physics sensitive to gluon polarization.

To achieve high polarization during collisions, RHIC is equipped with two helical dipole magnets known as snakes. Each snake rotates the spin direction of the protons by 180 degrees. This helps cancel the lower order field perturbations which can depolarize the beam and keeps the net spin precession rate per turn or spin tune $\left(\nu_{s}\right)$ energy independent. RHIC is also equipped with a pair of spin rotators located around each of the experiments (STAR and PHENIX). They are also constructed from helical dipoles. However they are designed to rotate the spin toward an arbitrary direction at the collision point. The layout of the accelerator complex is shown in Fig. 1.

RHIC typically achieves average polarization over single 6-8 hour store of $55 \%$. One of the important factors effecting the average proton polarization during a store is the polarization lifetime. Polarization lifetime is defined as the \% reduction of polarization per hour. Measured polarization lifetime in RHIC has varied between a low of

\footnotetext{
Corresponding author. vranjbar@bnl.gov

Published by the American Physical Society under the terms of the Creative Commons Attribution 4.0 International license. Further distribution of this work must maintain attribution to the author(s) and the published article's title, journal citation, and DOI.
}

about $0.17 \%$ per hour to a high of $3 \%$ per hour. However understanding polarization lifetime in the presence of orthogonal snakes is still challenging. We do not yet possess a good theory to explain polarization losses in this system, neither has the community been able to simulate these losses. For example direct spin tracking to simulate 1 hour of beam time would take 300 million turns in RHIC. A low resolution study using larger step sizes (4-8 slices per quad/dipole) would require approximately 1 hour to perform 100K RHIC turns using the spin-orbit code teaspink [1]. Zgoubi [2] another spin-orbit code has similar timescales. Thus to simulate 1 hour of beam time would take at least 125 days. Even if we would allocate the time we do not have the computer resources to do this for any kind of realistic distribution using existing direct tracking codes.

So in lieu of this, we have turned to the lattice independent spin tracking methods developed previously [3]. This approach involves integrating the ThomasBargmann-Michel-Telegdi (T-BMT) spin equation using

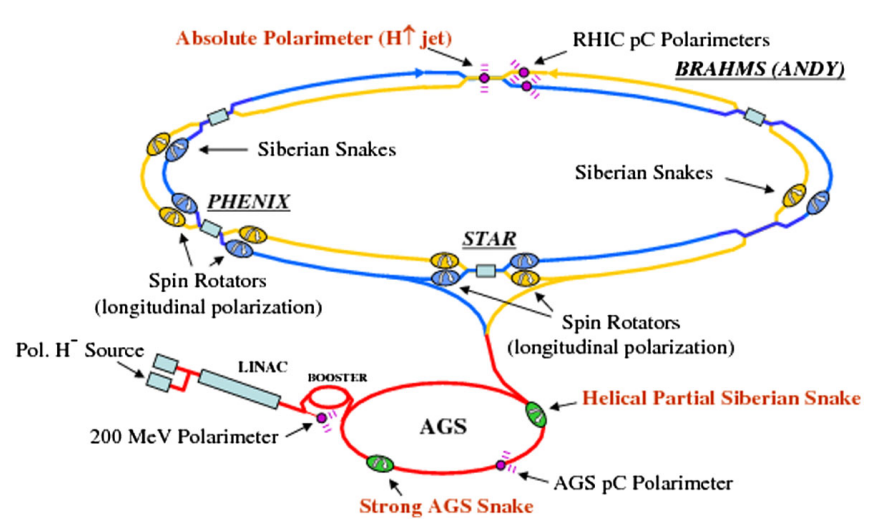

FIG. 1. RHIC-AGS complex. 
only several spin resonances with a unitary 4th order Gaussian quadrature integrator. This model, benchmarked against direct spin-orbit tracking codes, has proven very effective in simulating and studying polarization loss during the RHIC acceleration ramp. However when initially applied to consider issues of polarization lifetime, it could not generate polarization lifetime over $0.01 \%$ on the timescales of several hours of beam time in RHIC.

This situation changed when we extended the algorithm to include longitudinal dynamics. The introduction of longitudinal dynamics provided a mechanism for polarization lifetime on timescales similar to what was observed in RHIC. In this paper we present the results from this extension of the differential equation to handle longitudinal dynamics.

Using this model we studied the effect of several lattice parameters on polarization lifetime and found that the existence of longitudinal dynamics appears to be necessary to generate polarization lifetime on the timescales of 1 hour of beam time. However the effect of longitudinal dynamics is a purely threshold effect. Its existence is necessary for the observation of a finite polarization lifetime, but the details and values of associated parameters seem to play a very small role in polarization lifetime for realistic values used in RHIC. The most important factors in determining the magnitude of the losses are the strength of the spin resonances.

In addition we have also developed an analytical approach to study the effect of longitudinal dynamics in the T-BMT equation. We follow the approach outlined in [4] and consider the system in the parametric resonances formalism.

As we will discuss in the final sections, this model does not capture all of the physics associated with polarization lifetime. There are effects due to emittance growth and higher-order fields which such a simple model ignores. However this model does seem to capture most of the important first-order effects which dominate the causal factors for polarization lifetime.

\section{SPIN DYNAMICS REVIEW}

The dynamics of the spin vector of a charged particle with $q$ charge in the laboratory frame is described by the T-BMT equation,

$$
\frac{\mathrm{d} \vec{S}}{\mathrm{~d} t}=\frac{q}{\gamma m} \vec{S} \times\left[(1+G \gamma) \vec{B}_{\perp}+(1+G) \vec{B}_{\|}\right],
$$

$\vec{S}$ is the spin vector of a particle in the rest frame, and $\vec{B}_{\perp}$ and $\vec{B}_{\|}$are defined in the laboratory rest frame with respect to the particle's velocity. $G=\frac{g-2}{2}$ is the anomalous magnetic moment coefficient which for protons is 1.7928474 , and $\gamma m c^{2}$ is the energy of the particle. Here we neglect the electric fields. We can transform this equation by

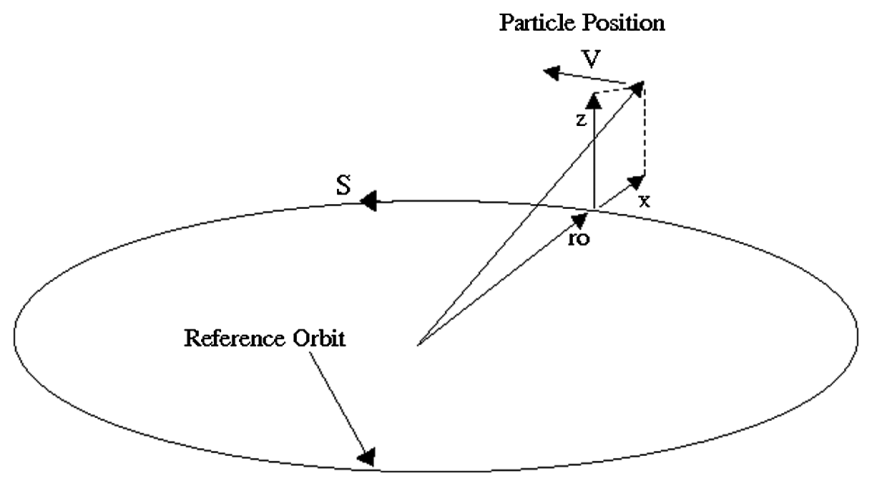

FIG. 2. The curvilinear coordinate system for a particle motion in a circular accelerator. $\hat{x}, \hat{s}$, and $\hat{z}$ are the transverse radial, the longitudinal, and the transverse vertical unit basis vectors, and $\vec{r}_{0}(s)$ is the reference orbit.

expanding about a reference orbit described by the Frenet-Serret coordinate system shown in Fig. 2. Thus we have

$$
\frac{\mathrm{d} \hat{x}}{\mathrm{~d} s}=\frac{\hat{s}}{\rho}, \quad \frac{\mathrm{d} \hat{s}}{\mathrm{~d} s}=-\frac{\hat{x}}{\rho}, \quad \text { and } \quad \frac{\mathrm{d} \hat{z}}{\mathrm{~d} s}=0
$$

where $\rho$ is the local radius of curvature for the reference orbit. This is satisfactory for a trajectory in the plane (no vertical bends). Particle motion can be parametrized in this coordinate system as,

$$
\vec{r}=\vec{r}_{o}(s)+x \hat{x}+z \hat{z} .
$$

Here, $\vec{r}_{o}(s)$ is the reference orbit, and $\hat{s}=\mathrm{d} \vec{r}_{o} / \mathrm{d} s$. Following a standard derivation [5] the fields of the T-BMT equation can be recast to first order in terms of the transverse coordinates $x, z$ and the local bending radius $\rho$ and their derivatives.

$$
\begin{aligned}
\frac{\mathrm{d} \vec{S}}{\mathrm{~d} s} & =\vec{f} \times \vec{S} \\
f_{1} & =(1+G \gamma) z^{\prime \prime} \\
f_{2} & =-(1+G \gamma) z^{\prime} / \rho+(1+G)\left(\frac{z}{\rho}\right)^{\prime} \\
f_{3} & =-x^{\prime \prime}(1+G \gamma)+\frac{G \gamma}{\rho} .
\end{aligned}
$$

Defining a two-component spinor $\Psi$ such that the $i$ th component of the spin vector is given by

$$
S_{i}=\left\langle\Psi\left|\sigma_{i}\right| \Psi\right\rangle=\Psi^{\dagger} \sigma_{i} \Psi
$$

The T-BMT equation becomes: 


$$
\frac{\mathrm{d} \Psi}{\mathrm{d} s}=\frac{i}{2}(\vec{\sigma} \cdot \vec{f}) \Psi=\frac{i}{2} H \Psi=-\frac{i}{2}\left(\begin{array}{cc}
\frac{G \gamma}{\rho} & -\zeta(s) \\
-\zeta(s)^{*} & -\frac{G \gamma}{\rho}
\end{array}\right) \Psi .
$$

Here we have dropped $-x^{\prime \prime}(1+G \gamma)$ the first term in $f_{3}$ since it is a small term compared to $G \gamma$. However this term can generate a set of important hybrid resonances around the sidebands of dominant spin resonances [6]. Also $\vec{\sigma}=\left(\sigma_{x}, \sigma_{s}, \sigma_{z}\right)$ is a vector of the Pauli spin matrices and $\zeta(s)=-f_{1}+i f_{2}$. Equation (6) is a form of the Schroedinger equation, however $\Psi$ is here a classical vector in which components, $u$ and $d$, are related to the 3 components of $S$ by

$$
\begin{aligned}
& S_{1}=u^{*} d+u d^{*} \\
& S_{2}=-i\left(u^{*} d-u d^{*}\right) \\
& S_{3}=|u|^{2}-|d|^{2} .
\end{aligned}
$$

Since $H$ is Hermitian the magnitude of the spin vector

$$
|\vec{S}|=|u|^{2}+|d|^{2}=\Psi^{\dagger} \Psi
$$

remains constant. We chose the normalization condition for the spinor function to be $\Psi \dagger \Psi=1$.

The standard approach used in past books [7] and papers by several authors including myself has been to follow Courant and Ruth's technical note [5]. Here Eq. (6) is transformed by moving from $s$ as the independent variable to the turning angle $\Theta=\int_{0}^{s} \frac{\mathrm{d} s}{\rho}$. $\Theta$ is usually equivalent to the dipole bending angle but properly it is the spin precession about the vertical axis. In this case $\zeta$ becomes a function of $\Theta$ which is expanded in a Fourier series:

$$
\zeta(\Theta)=\sum_{K} \mathrm{w}_{K} e^{-i K \Theta} .
$$

Here $K$ is the spin resonance frequency and $\mathrm{w}_{K}$ is the strength of the resonance at $K=G \gamma$. However there has been some discussion that this approach might mishandle the combined precessions about the horizontal and longitudinal axis caused by quadrupole and solenoid fields in the straight sections where $\rho=\infty$ and the turning angle does not advance. Ordinarily rotations about different axes should not commute although here they are differentiated by the fact that precessions about the horizontal are real and those about the longitudinal axis are imaginary. Additionally since we want to move to a differential equation which is continuous to handle a continuous approximation of the transverse phase effects due to longitudinal dynamics, we have chosen to instead follow a derivation developed later by Courant [8]. This approach avoids any discontinuity in the differential equation which the introduction of the turning angle as an independent variable might cause. Instead it uses the average orbital angle $\theta=\frac{s}{R}$ with $R$ equal to the average radius as the independent variable and further Fourier expands in this variable. Using the average orbital angle also maintains some consistency with Hoffstaetter's book [9] which also uses the average orbital angle. Fortunately the new approach does not alter the ultimate DEPOL algorithm used to calculate the spin resonance strength developed in [5].

Using this new approach we can transform Eq. (6) into a frame which beats with the turning angle $\Theta$ and the averaged orbital angle $\theta$. $\Theta$ advances only in the dipoles giving $\mathrm{d} \Theta=\mathrm{d} s / \rho$. In the straight sections where $\rho=\infty$ the change in orbital angle is zero $(\mathrm{d} \Theta=0)$.

$$
\begin{aligned}
& \Phi=e^{\frac{i}{2} \chi \sigma_{z} \Psi} \\
& \chi=G \gamma(\theta-\Theta) .
\end{aligned}
$$

The BMT equation now becomes:

$$
\frac{\mathrm{d} \Phi}{\mathrm{d} s}=-\frac{i}{2}\left(\begin{array}{cc}
\frac{G \gamma}{R} & -\zeta e^{-i \chi} \\
-\zeta^{*} e^{i \chi} & -\frac{G \gamma}{R}
\end{array}\right) \Phi .
$$

Ignoring the effects of longitudinal motion, one can evaluate the cumulative effect of the lattice on the spin, by expanding $\zeta e^{-i \chi}$ in the average orbital angle $\theta$

$$
\zeta e^{-i \chi}=\frac{1}{R} \sum_{K} \mathrm{w}_{K} e^{-i K \theta} .
$$

In the case of closed orbit errors, imperfection spin resonances will occur when $K=$ integer and the strength can be calculated using:

$$
\mathrm{w}_{K}=\frac{-1}{2 \pi} \int_{0}^{2 \pi R} \zeta e^{i(K \theta-\chi)} \mathrm{d} s .
$$

If there are betatron oscillations present, then intrinsic spin resonances will occur when $K=k \pm Q$ where $Q$ is the betatron tune and $k$ is an integer. In this case the Fourier integral can involve an irrational $K$ values and it is properly evaluated over an infinite number of turns,

$$
\mathrm{w}_{K}=\lim _{N_{T} \rightarrow \infty} \frac{-1}{2 \pi N_{T}} \int_{0}^{L N_{T}} \zeta e^{i(K \theta-\chi)} \mathrm{d} s .
$$

Or $m$ turns if $K=n / m$ or one turn if $K$ is an integer. Even in the irrational case it can be possible for the integral to be evaluated using only one turn due to cancelation of terms and the ability to factor out the positive and negative betatron frequency components. Here, $L=2 \pi R$ is the path length around the lattice. If evaluated when $G \gamma=K$ this returns us to the original DEPOL [5] formulation: 


$$
\begin{aligned}
\mathrm{w}_{K}= & \lim _{N_{T} \rightarrow \infty} \frac{-1}{2 \pi N_{T}} \int_{0}^{L N_{T}}\left[(1+G \gamma)\left(z^{\prime \prime}+\frac{i z^{\prime}}{\rho}\right)\right. \\
& \left.-i(1+G)\left(\frac{z}{\rho}\right)^{\prime}\right] e^{i K \Theta(s)} \mathrm{d} s .
\end{aligned}
$$

The calculation of Eq. (15) has been implemented in a program called DEPOL [5] which has been used for many decades to evaluate spin resonances in the AGS and RHIC machines. A version to handle the case of betatron coupling due to skew quadrupole fields and solenoidal magnets was developed later [10]. There also exists a code called SPRINT [11] which can perform this calculation for an arbitrary spin orientation (i.e., stable spin direction nonvertical) based on the code SLIM [12] and using the approach outlined in [9].

It is convenient to write Eq. (6) in terms of $\theta$,

$$
\frac{\mathrm{d} \Phi}{\mathrm{d} \theta}=-\frac{i}{2}\left(\begin{array}{cc}
G \gamma & -\hat{\zeta}(\theta) \\
-\hat{\zeta}(\theta)^{*} & -G \gamma
\end{array}\right) \Phi
$$

Here, $\hat{\zeta}(\theta)=\sum_{K} \mathrm{w}_{K} e^{-i K \theta}$ and for a single resonance term in $\mathrm{w}_{K} e^{-i K \theta}$ Eq. (16) can be cast as a homogeneous second order differential equation (see e.g., [4]). In the nonaccelerating case ( $G \gamma=$ constant) this differential equation has only constant coefficients and thus the solution is like a damped harmonic oscillator. In the accelerating case $\left(G \gamma=G \gamma_{0}+\alpha \theta\right)$ the coefficients are functions of $\theta$, and solutions can be found following Froissart-Stora [13], given by the confluent hypergeometric functions. In the asymptotic limit of $\theta \rightarrow \pm \infty$ these functions have simpler forms. Using this, the amount of depolarization caused by acceleration through any given single spin resonance can be evaluated with the famous Froissart-Stora formula,

$$
\frac{P_{f}}{P_{i}}=2 e^{-\left(\pi\left|\mathrm{w}_{K}\right|^{2} / 2 \alpha\right)}-1,
$$

where,

$$
\alpha=\frac{1}{\omega_{\text {rev }}} \frac{d \nu_{s}}{\mathrm{~d} t}
$$

is the rate of change of the spin tune divided by the angular revolution frequency $\omega_{\text {rev }}$, and $\frac{P_{f}}{P_{i}}$ is the ratio of final vertical to initial vertical polarization. For a flat orbit in a constant vertical field $\alpha \approx \mathrm{d}(G \gamma) / \mathrm{d} \theta$.

\section{LATTICE INDEPENDENT INTEGRATION}

We have previously developed a code to integrate the $2 \mathrm{D}$ spinor form of the T-BMT equation [Eq. (16)] [3]. Using a 4th order Magnus Gaussian quadrature integrator described in [14] we can integrate Eq. (16) for an arbitrary $\hat{\zeta}(\theta)$. In this code the effect of snakes are handled separately and are added at the appropriate place in $\theta$ as optically transparent thin spin kicks using the matrix,

$$
T_{s}=e^{-i \frac{\pi}{2} \hat{n}_{s} \cdot \vec{\sigma}}
$$

with $n_{s}=\hat{e}_{1} \cos \phi_{s}+\hat{e}_{2} \sin \phi_{s}$ and $\phi_{s}$ the snake angle.

However as long as the system was held away from a low order snake resonance, integrating this system with any combination of intrinsic and imperfection resonances over 600 million turns showed no mechanism for polarization loss greater than $0.01 \%$.

So we introduced the effect of longitudinal motion into our system. The effects of longitudinal motion on transverse betatron motion have been studied and derived in several standard textbooks (e.g., see [7]) using the sychrobetatron Hamiltonian. However since we are analyzing a reduced "smoothed" system, a smooth approximation using Hills equation is easier and more appropriate. To understand the effects of longitudinal motion on spin we need to first understand its effect on transverse motion. In the simplest case a single particle's vertical motion without longitudinal effects can be characterized by

$$
\frac{\mathrm{d}^{2} z(s)}{\mathrm{d} s^{2}}+\frac{\omega_{\beta}^{2}}{c^{2}} z(s)=0
$$

Here $c$ is the speed of light and $\omega_{\beta}$, the angular betatron frequency, is the angular revolution frequency $\omega_{0}$ times $Q$ the betatron tune. Solutions give transverse harmonic motion oscillating with the betatron tune $Q$ each revolution. However if the particle resides in a rf-bucket one must consider its longitudinal motion inside of the bucket as well and the equation of motion becomes,

$$
\frac{\mathrm{d}^{2} z(s ; \delta, \tau)}{\mathrm{d} s^{2}}+\frac{\omega_{\beta}^{2}[\delta(s)]}{c^{2}} z\left(s ; \delta_{0}, \tau_{0}\right)=0 .
$$

Here $\tau$ defines the time difference relative to the center of the rf-bucket. Here $\tau_{0}=\tau(0)$ or the initial $\tau(s)$ value. It can also be expressed in terms of the longitudinal position $(\sigma)$ in the rf-bucket $\tau=\sigma / c . \delta=\Delta p / p$ is the relative momentum difference from the on momentum particle. Here $\delta_{0}=\delta(0)$ or the initial $\delta(s)$ value. If we expand the betatron frequency to first order in $\delta$ we obtain,

$$
\omega_{\beta}[\delta(s)]=\omega_{0} Q+\xi \omega_{0} \delta(s)
$$

where $\xi=\frac{\mathrm{d} Q}{\mathrm{~d} \delta}$ is the linear chromaticity. We can also approximate the longitudinal motion inside the rf-bucket using, 


$$
\begin{aligned}
\delta(s) & =\frac{-\omega_{s}}{\eta c} r \sin \left(\omega_{s} s / c+\phi\right) \\
\tau(s) & =r c \cos \left(\omega_{s} s / c+\phi\right) .
\end{aligned}
$$

Here $\omega_{s}=\omega_{0} Q_{s}$ is the synchrotron angular frequency, $\phi$ is the phase of the synchrotron motion, $\eta$ the phase-slip factor, defined as the fractional change of the revolution period per unit of $\delta$. Following [15,16,17], an approximate solution to Eq. (21) has the form,

$$
z(s ; \delta, \tau) \approx A e^{ \pm i \Phi(s ; \delta, \tau)}
$$

where $A$ is the constant amplitude and,

$$
\begin{aligned}
\Phi(s ; \delta, \tau)= & \int_{0}^{s} \mathrm{~d} s^{\prime}\left[\omega_{0} Q / c+\omega_{0} \xi \delta\left(s^{\prime}\right) / c\right] \\
= & \omega_{0} Q s / c+\frac{\xi \omega_{0}}{\eta}[\tau(s)-\tau(0)] \\
= & \omega_{0} Q s / c+\frac{\xi \omega_{0} \tau(0)}{\eta}\left[\cos \left(\omega_{s} s / c\right)-1\right] \\
& +\frac{\xi \delta(0)}{Q_{s}} \sin \left(\omega_{s} s / c\right) .
\end{aligned}
$$

Here we recast the phase in terms of initial $\tau(0)$ and $\delta(0)$ using the transformation from the frame rotating with the synchrotron frequency,

$$
\left(\begin{array}{l}
\tau \\
\delta
\end{array}\right)(s)=\left(\begin{array}{cc}
\cos \left(\omega_{s} s / c\right) & \frac{\eta}{\omega_{s}} \sin \left(\omega_{s} s / c\right) \\
-\frac{\omega_{s}}{\eta} \sin \left(\omega_{s} s / c\right) & \cos \left(\omega_{s} s / c\right)
\end{array}\right)\left(\begin{array}{l}
\tau \\
\delta
\end{array}\right)(0) .
$$

The result of the longitudinal motion is the addition of a betatron phase modulating term which oscillates with the synchrotron frequency. The modulation of the betatron phase as function of $\tau(0)$ has been measured extensively and has actually been employed to measure the linear chromaticity of a lattice at the SPS, Tevatron and RHIC machines $[15,18,19]$.

Writing $\tau(0)$ as $\tau_{0}$ and moving from $s$ to angle $\theta$ using $\omega_{s} s / c=Q_{s} \theta$, we can now model the effect of longitudinal motion via a phase modulation term $g(\theta)=$ $e^{i \frac{\xi \omega_{0} \tau_{0}}{\eta}\left[1-\cos \left(Q_{s} \theta\right)\right]}$. This betatron phase modulation will effect the spin resonance by modulating its betatron phase terms in $\hat{\zeta}(\theta)$. The betatron phase terms for the spin resonances are contained in the complex phase of the $\mathrm{w}_{K}=a_{k} e^{-i \phi_{K}}$ terms. Here $a_{k}$ is the magnitude of the resonance $\left|\mathrm{w}_{K}\right|$ and $\phi_{K}$ the complex phase which is due to the betatron phase. So one needs only add the modulated phase to the complex phase.

Thus to model the longitudinal effect on the spin resonances we multiply our $g(\theta)$ term by the $\hat{\zeta}(\theta)$ term [we now call $\zeta_{0}(\theta)$ ] to obtain a new $\hat{\zeta}$,

$$
\begin{aligned}
\hat{\zeta}(\theta) & =\zeta_{0}(\theta) g(\theta) \\
& =\sum_{K} \mathrm{w}_{K} e^{-i K \theta+i \frac{\xi \omega_{0} \tau_{0}}{\eta}\left[1-\cos \left(Q_{s} \theta\right)\right]} .
\end{aligned}
$$

Here the initial betatron phase is absorbed into the complex phase of $\mathrm{w}_{K}=a_{K} e^{-i \phi_{K}}$. We choose the initial $\delta(0)=0$ since that will only alter the constant initial phase and should not contribute to the dynamics which drive polarization lifetime.

In addition to the phase effect there is also a direct energy modulating effect which has previously been described by Lee and Berglund [20]. This effect modifies $G \gamma$ as follows,

$$
G \gamma(\theta)=\left(G \gamma_{0}+\alpha \theta\right)[1+\delta(\theta)] .
$$

Here we have left out the relativistic $\beta^{2}$ since for the regimes we are interested in it is $\approx 1$. Because we have chosen $\delta(0)=0$, in terms of $\theta, \delta(\theta)=\omega_{s} \tau_{0} \sin \left(Q_{s} \theta\right) / \eta$, we track assuming all particles have the same initial emittance with an evenly distributed betatron phases $\phi_{K}$ between 0 and $2 \pi$. For each tracking we give $\omega_{0}, \omega_{s}, \eta, C_{\delta}$ and to simulate the effects of different beam actions we give $\tau_{0}$ and $a_{k}$ for each grouping of $K$ resonances considered. Our actual implementation updates $g(s)$ only once per turn since the timescale of the synchrotron motion is on the order of 100's of turns.

\section{NUMERICAL INTEGRATION SETUP}

Our code was set up to integrate Eq. (16) with $\hat{\zeta}(\theta)$ given by:

$$
\hat{\zeta}(\theta, m)=\sum_{K} \mathrm{w}_{K} e^{-i K \theta+i \frac{\xi \omega_{0} \tau_{0}}{\eta}\left[1-\cos \left(2 \pi Q_{s} m\right)\right]} .
$$

Here $m$ is the turn number and $\mathrm{w}_{K}$ are evaluated using Eq. (15). The phase due to the synchrotron motion is updated only once per turn as is the value of $G \gamma$. Prior to integration Eq. (16) was transformed to the interaction frame using $U=e^{\frac{-i}{2} G \gamma \theta \sigma_{z}}$. The new equation written in matrix form, with its initial conditions is,

$$
\Psi^{\prime}=A(\theta) \Psi, \quad \Psi\left(\theta_{0}\right)=\Psi_{0} .
$$

The 4th order Magnus Gaussian quadrature integrator evaluates the matrix $A(\theta)$ at two orbital locations $\theta+\left(\frac{1}{2} \pm \frac{\sqrt{3}}{6}\right) h$ with step size $h$ :

$$
\begin{aligned}
& A_{1}=A\left[\theta_{n}+\left(\frac{1}{2}-\frac{\sqrt{3}}{6}\right) h\right] \\
& A_{2}=A\left[\theta_{n}+\left(\frac{1}{2}+\frac{\sqrt{3}}{6}\right) h\right] .
\end{aligned}
$$


These are then used to calculate the $\Omega$ used to propagate the spinor:

$$
\begin{aligned}
\Omega^{[4]}(h) & =\frac{h}{2}\left(A_{1}+A_{2}\right)-h^{2} \frac{\sqrt{3}}{12}\left[A_{1}, A_{2}\right] \\
\Psi_{n+1} & =\exp \left(\Omega^{[4]}(h)\right) \Psi_{n} .
\end{aligned}
$$

Here we use the identity,

$$
\exp (i \vec{a} \cdot \vec{\sigma})=\cos (|a|) I+i \frac{\sin (|a|)}{|a|} \vec{a} \cdot \vec{\sigma}
$$

to evaluate the matrix exponential, where $|a|=$ $\sqrt{a_{1}^{2}+a_{2}^{2}+a_{3}^{2}}$. We decompose our $\Omega$ into $\vec{a} \cdot \vec{\sigma}$ using $a_{1}=$ $\Im\left(\Omega_{12}\right), a_{2}=\Re\left(\Omega_{12}\right), a_{3}=\Im\left(\Omega_{11}\right)$ to apply Eq. (33). In our case $h=\pi / 20$ was used yielding a total of 40 steps from $\theta 0$ to $2 \pi$. At $\theta=\pi$ and $2 \pi$ a snake spin transport matrix given by Eq. (19) is applied with $\phi_{s}= \pm \pi / 4$. The code is available at [21].

\section{NUMERICAL ERROR CHECK}

Since we are running our integrator over $10^{8}$ turns one important concern is the development of roundoff errors and deviations from unitarity. Checks of unitarity found deviations of less than $1 \times 10^{-8}$ after tracking over two hours. We also performed reverse tracking. For 128 particles tracked over 1 hour $\left(2.5 \times 10^{8}\right.$ turns $)$ reverse tracking recovered the starting spin values to within $5 \times 10^{-6}$ (see Fig. 3).

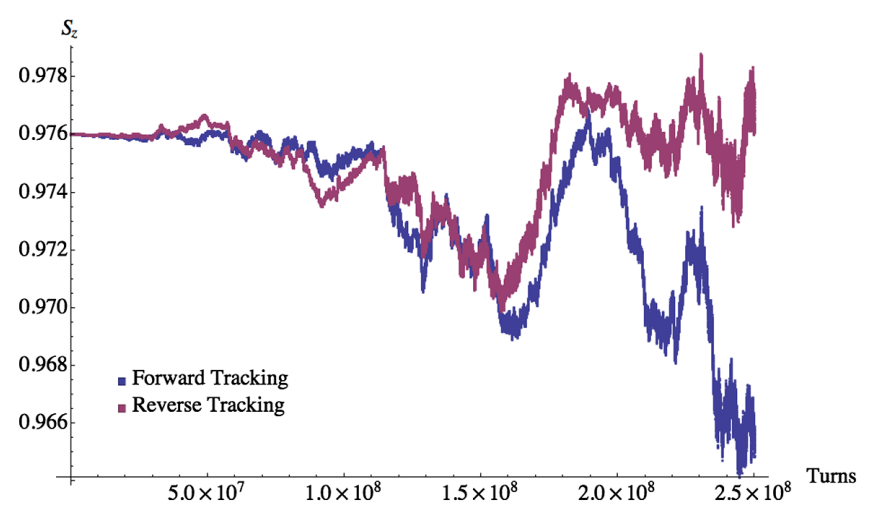

FIG. 3. Average evolution of polarization for forward and reverse tracking for 1 hour (1 hour forward, 1 hour reverse). Path's diverged initially because reverse tracking is staggered by one turn and printing is done every 2000 turns. We track at $G \gamma=191.5$ including a single resonance at $K=191.693$ with $\mathrm{w}_{K}=0.017646+0.054524 i$. Other beam parameters are $Q=30.693, C_{\delta}=2.0, Q_{s}=8.9 \times 10^{-4}, \eta=0.001566$ and $\tau_{0}=0.1 \mathrm{~ns}$. Note $S_{z}=0.976$ is the vertical $(z)$ component of stable spin direction for this system calculated via stroboscopic averaging.

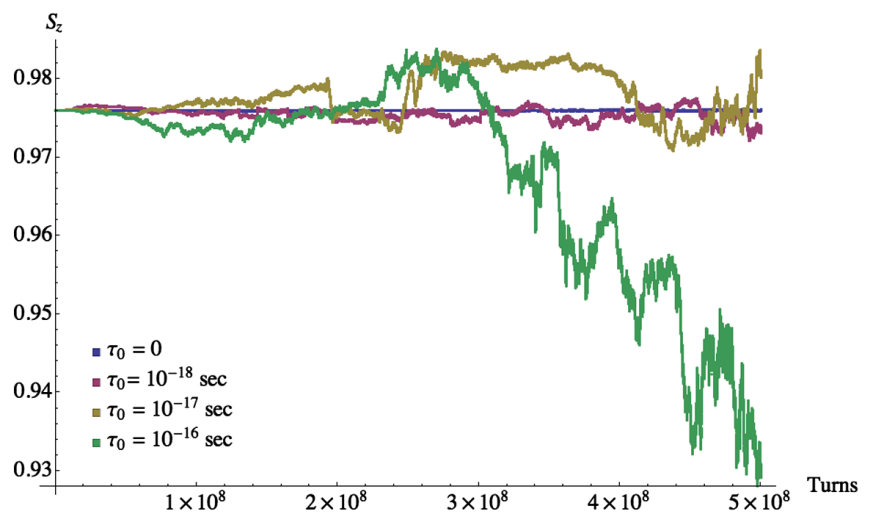

FIG. 4. Turn-by-turn average evolution of polarization for different initial $\tau_{0}$ in presence of a single resonance with two orthogonal snakes. All other beam parameters are same as in Fig. 3. Note we also considered $\tau_{0}=10^{-15}$ but did not plot it as it overlaid the $10^{-16}$ trace.

\section{POLARIZATION LIFETIME AND ITS CAUSES}

As can be seen in Fig. 4 the integration of the T-BMT equation for a single resonance with two orthogonal snakes, over 2 hours of beam time, show no observable polarization loss for $\tau_{0}=0$. The introduction of longitudinal motion however changes this situation very dramatically. Additionally the threshold appears to be less than $10^{-18} \mathrm{sec}$ in $\tau_{0}$ amplitude $\left(\hat{\delta} \approx 2.8 \times 10^{-13}\right)$ since that is the smallest $\tau_{0}$ value we checked and still observed polarization decay on the hour timescale. However above $\tau_{0}=10^{-16} \sec \left(\hat{\delta} \approx 2.8 \times 10^{-11}\right)$ the decay became more pronounced assuming a level of polarization loss fairly constant out to as high as $\tau_{0}=10^{-8} \sec \left(\hat{\delta} \approx 2.8 \times 10^{-3}\right)$. In the presence of six additional nearby intrinsic resonances we see that like the single resonance case, it is only with the introduction of longitudinal motion that a discernable polarization decay appears on the timescales of 1 hour (see Fig. 5). Concerning the response of the polarization lifetime to different factors related to longitudinal motion, there are several noticeable features. First although the overall polarization lifetime is rather insensitive to the magnitude of synchrotron tune, chromaticity and longitudinal amplitude they do provide for a larger spread in the turn-by-turn average polarization.

So for example we saw that with only $10^{-16} \mathrm{sec}$ timing offset from the bucket center polarization decay sets in. But above this, changes of initial $\tau_{0}$, synchrotron tune and chromaticity show little correlation with increased decay rates, but they do demonstrate larger polarization spread (see Fig. 6). While longitudinal dynamics seems essential to trigger the process of polarization decay, the response is rather insensitive to changes in longitudinal parameters. In the range of reasonable values for synchrotron tune, momentum offset and chromaticity, decay rates do not change significantly. 


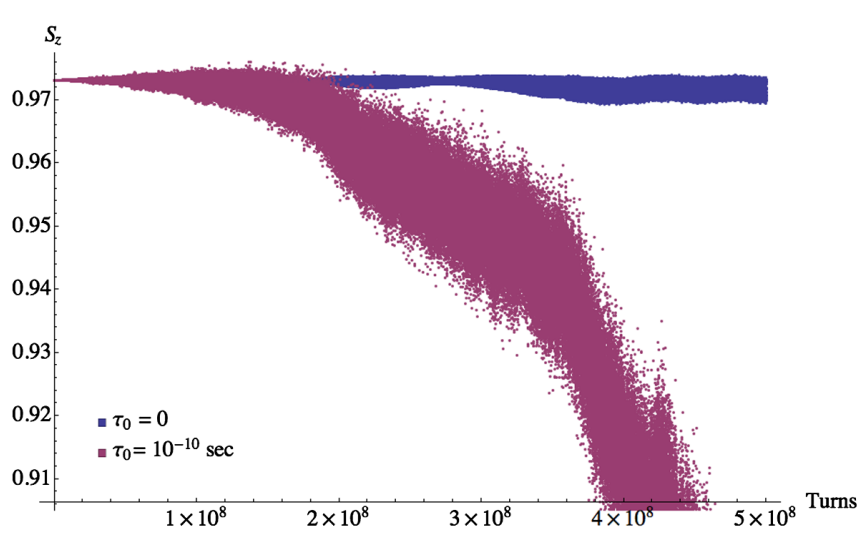

FIG. 5. Turn-by-turn average evolution of polarization for beam stored at $G \gamma=487$ with different initial $\tau_{0}$ including the effects of six neighboring resonances: $K_{1}=486.309, K_{2}=487.309$, $K_{3}=488.309, K_{4}=486.691, K_{5}=487.691$, and $K_{6}=488.691$. Here the strengths given by, $\mathrm{w}_{1}=0.018036+0.063010 i$, $\mathrm{w}_{2}=-0.000942-0.002246 i, \quad \mathrm{w}_{3}=0.005438-0.001752 i$, $\mathrm{w}_{4}=0.002344-0.006428 i, \mathrm{w}_{5}=0.005042+0.001384 i$, and $\mathrm{w}_{6}=-0.028228-0.009498 i$. These strengths are twice the DEPOL calculated value for $10 \mathrm{~mm}$-mrad normalized emittance. This translates into a particle with $40 \mathrm{~mm}$-mrad normalized emittance. For example see Eq. (34).

However polarization lifetime is very sensitive to the magnitude of the nearby intrinsic spin resonances. Here both proximity and strength play a role. Although the generally the proximity of the resonance is important, it appears that numerology is also a factor. In Fig. 7 we simulated the effects of a single spin resonance on beam stored at different energies. These simulations are performed by varying the location and strength of an intrinsic resonance. These were done using fractional tunes of 0.683 and 0.693 . We can see how for each resonance the polarization loss is proportional to the strength $|w|$. This maps out a polarization response curve for each resonance location. However the polarization losses are not always a direct function of the distance to the resonance. This in part is probably due to our tune's proximity to the 0.7 odd order snake resonance. There are probably other factors having to do with moving in and out of a parametric resonance region which will be discussed later.

Next we computed the response to various beam emittances. In the real machine there are several nearby intrinsic spin resonance. We used DEPOL [5] to calculate the strengths of the six nearest intrinsic spin resonance at $100 \mathrm{GeV}(G \gamma=191.5)$. Since the intrinsic spin resonance is proportional to the square root of the normalized emittance we can rescale an intrinsic spin resonance at one emittance to an arbitrary emittance using:

$$
\begin{aligned}
|\mathrm{w}(\epsilon)|^{2} & =\frac{\epsilon}{\epsilon_{c}} \cdot\left|\mathrm{w}\left(\epsilon_{c}\right)\right|^{2} . \\
\epsilon_{c} \frac{|\mathrm{w}(\epsilon)|^{2}}{\left|w\left(\epsilon_{c}\right)\right|^{2}} & =\epsilon
\end{aligned}
$$
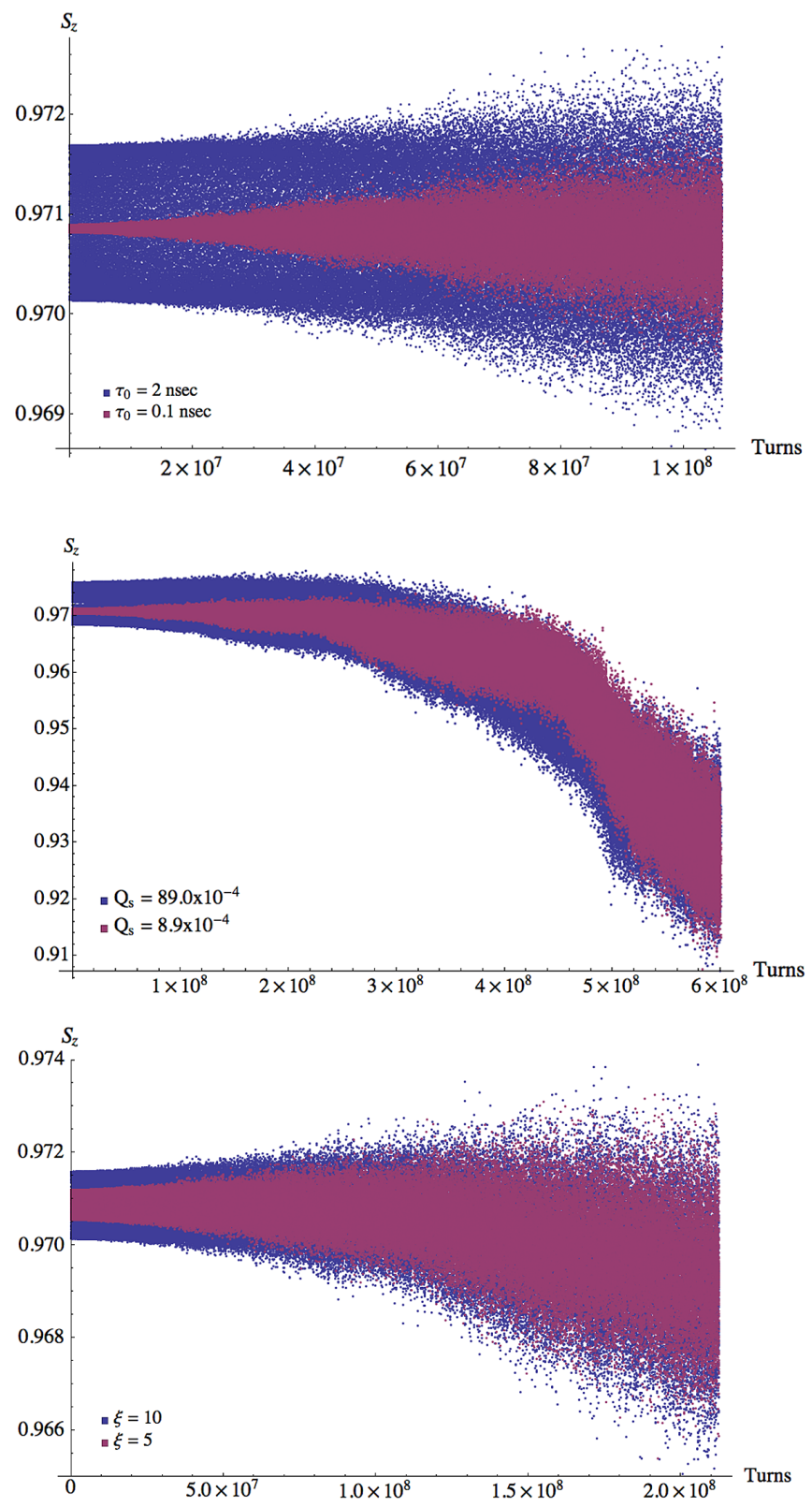

FIG. 6. Turn-by-turn average polarization for two different $\tau_{0}$ values with $Q_{s}=8.9 \times 10^{-4}$, chromaticity $=2.0$ (top). Turnby-turn average evolution of polarization for initial synchrotron tune $Q_{s}=8.9 \times 10^{-4}$ and $89.0 \times 10^{-4}$ with $\tau_{0}=5.0 \mathrm{nsec}$ and chromaticity $=2.0$ (middle). Turn-by-turn evolution for chromaticity $=5$ and 10 with $Q_{s}=8.9 \times 10^{-4}$ and $\tau_{0}=5.0$ nsec (bottom). The computations are all performed for stored $G \gamma=191.5$ and vertical tune of 30.693 with four intrinsic resonances calculated from the FY15 $100 \mathrm{GeV}$ pp lattice. We included six resonances $K_{1}=191.693, K_{2}=190.307, K_{3}=$ 192.307, $K_{4}=190.693, K_{5}=191.307$, and $K_{6}=192.693$. The associated DEPOL calculated strengths at $10 \mathrm{~mm}$-mrad normalized emittance for this lattice are $\mathrm{w}_{1}=-0.000095-0.001703 i$, $\mathrm{w}_{2}=-0.006119-0.007888 i, \quad \mathrm{w}_{3}=0.000000+0.001605 i$, $\mathrm{w}_{4}=-0.000029+0.000269 i, \mathrm{w}_{5}=0.008823+0.027262 i, \mathrm{w}_{6}=$ $0.000646-0.001524 i$. These were all scaled up to reflect a $40 \mathrm{~mm}$-mrad normalized emittance. For example see Eq. (34). 


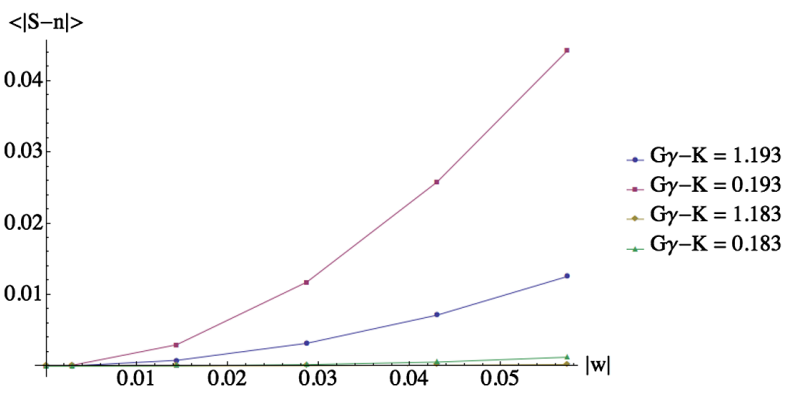

FIG. 7. Polarization deviation from stable spin direction after 1.77 hours at stored $G \gamma=191.5$, with $Q_{s}=8.9 \times 10^{-4}$ and $\tau_{0}=0.1 \mathrm{nsec}$. We consider the effects for a single intrinsic resonance. We compare the $K=221-Q_{y}$ and the $K=222-$ $Q_{y}$ resonance for two different tunes 30.693 and 30.683. This gives four lines at $K=190.307,191.307,190.317$, and 191.317. We plot against $|\mathrm{w}|$.

Here $\epsilon_{c}$ is the emittance of the calculated intrinsic spin resonance. In our case they were calculated at $10 \mathrm{~mm}$-mrad normalized emittance. Then for our simulations we multiplied all the resonances by a scale factor $S$ to evaluate the effect of different emittances. To recover the emittances we can take $S^{2} 10 \mathrm{~mm}$ mrad to obtain our $\mathrm{mm}$ mrad normalized value. In Fig. 8 we show depolarization versus emittance for different $\tau_{0}$ values. In addition to the intrinsic spin resonance, the effects of imperfection spin resonances can be considered. In this case the imperfection spin resonance strength are independent of emittance, so their magnitude is held fixed, while the intrinsic's strength are scaled. The result of a study for the RHIC lattice are shown in Figs. 9 and 10. In Fig. 9 a study for $255 \mathrm{GeV}$ is shown where the nearest imperfection resonance is at $G \gamma=487$. In Fig. 10 a study for 255 is plotted comparing with those for $100 \mathrm{GeV}$. For the $100 \mathrm{GeV}$ case the nearest are at $G \gamma=191$ and 192 (we picked 191) are shown. In all cases the effects of the imperfection resonance on polarization

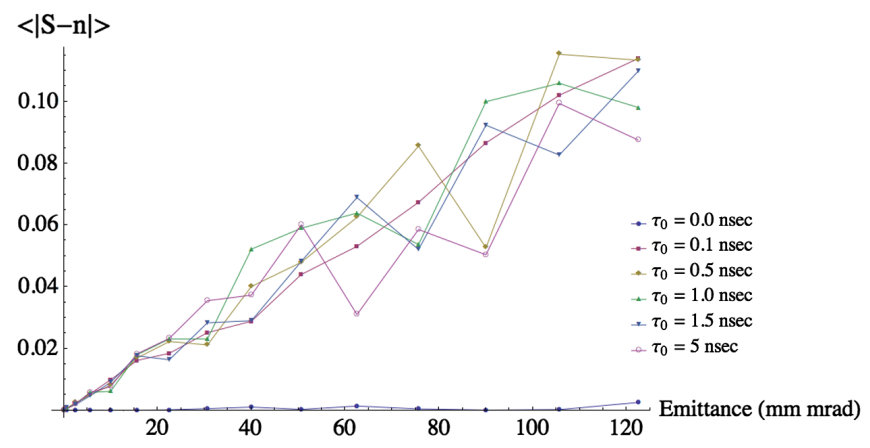

FIG. 8. Polarization deviation from stable spin direction after 1.77 hours at stored $G \gamma=191.5$, with $Q_{s}=8.9 \times 10^{-4}$. We consider the combined effects of the 6 nearby intrinsic resonances. These are shown for different $\tau_{0}$ plotted against particle emittance.

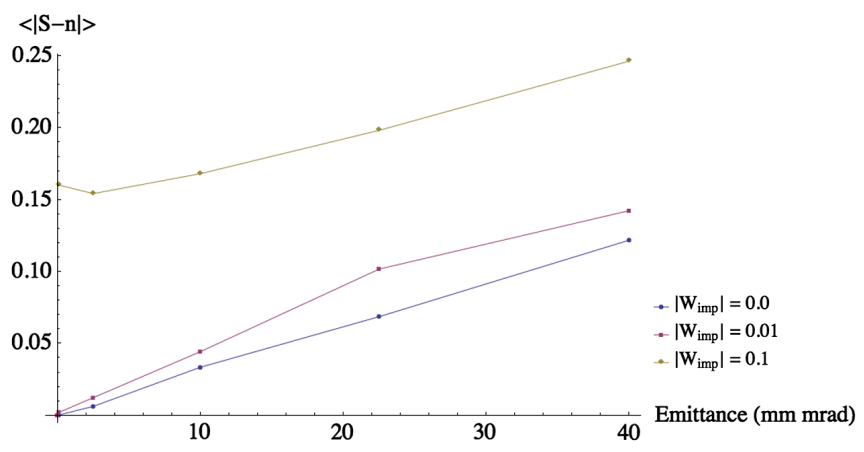

FIG. 9. Polarization deviation from stable spin direction after 1.77 hours for different initial imperfection resonances for $G \gamma=487$. Here the vertical tune was held at 29.691 with six intrinsic resonances calculated from the FY17 RHIC $255 \mathrm{GeV}$ lattice located at $K=486.309,487.309$, 488.309, 486.691, 487.691, and 488.691.

lifetime manifest themselves above 0.01 with significant losses at 0.1. Using the curves similar to those shown in Figs. 6 and 7 one can provide an estimate for the polarization lifetime by interpolating loss versus emittance curves and then integrating over the emittance distribution. Assuming a Gaussian distribution,

$$
\rho(\epsilon)=\frac{e^{-\frac{\epsilon}{2 \epsilon_{0}}}}{2 \epsilon_{0}}
$$

one obtains,

$$
\mathrm{Pol}_{\text {loss }}=\int_{0}^{\epsilon_{\max }} \mathrm{d} \epsilon \rho(\epsilon) f(\epsilon)
$$

Here $f(\epsilon)$ is a fit of the polarization loss versus emittance curves. In Fig. 10 we plot the estimated polarization lifetimes using this approach for both the FY15 and FY17 lattices. From this we can see that $f(\epsilon)$ should be well approximated by a linear function:

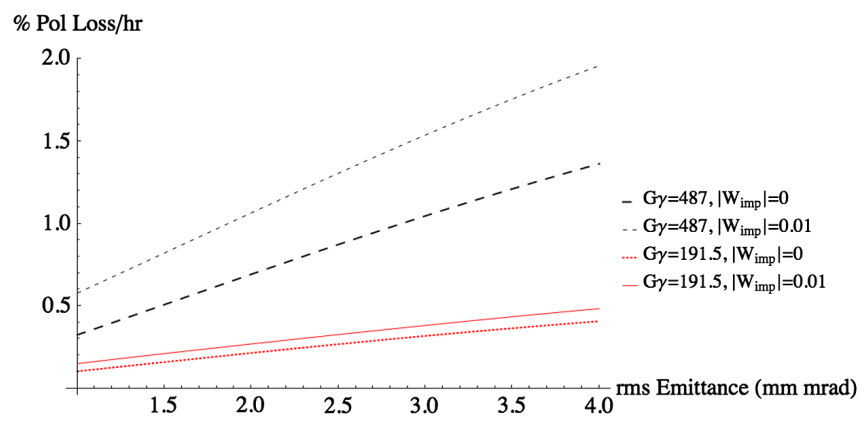

FIG. 10. Polarization loss per hour versus rms beam emittance for FY15 $100 \mathrm{GeV}$ lattice $(G \gamma=191.5)$ and the FY17 $255 \mathrm{GeV}$ lattice $(G \gamma=487)$. 


$$
f(\epsilon)=m \epsilon+b .
$$

Equation (36) can now be evaluated to give:

$$
\mathrm{Pol}_{\text {loss }}\left(\epsilon_{0}, \epsilon_{\mathrm{max}}\right)=b+2 \epsilon_{0} m-\frac{m \epsilon_{\mathrm{max}}}{e^{\frac{\epsilon_{\max }}{2 \epsilon_{0}}}-1} .
$$

Using the linear fits to the plots like those shown in Fig. 10 we can extract our slope $(m)$ and constant $(b)$ to estimate the lifetime for different emittances.

\section{COMPARISON WITH MEASURED DATA}

In Table I we show the measured lifetimes from four years of RHIC operations. These include FY12, FY13, FY15, and FY17 RHIC runs. This data is derived from [22] described in [23]. These results show that the rotators can have a detrimental effect on polarization lifetime. Especially if we consider the case of FY15 when the same lattices were run with and without the rotators. The rotators can have an effect identical to an imperfection spin resonance via two mechanisms. The first is if the rotators fail to be spin transparent and introduce a spin kick. This error in the rotation angle is given by,

$$
\mathrm{w}_{\mathrm{eff}}=\frac{\Delta \phi}{\pi} .
$$

So for example an 18 degree error will yield 0.1 imperfection spin resonance.

Additionally our experience in adjusting the currents in the spin rotators have shown that there is a significant effect on the orbit from the rotators. This change in orbit can effect the strength of the nearest imperfection spin resonance. It has also been observed that the orbit can in turn effect the spin orientation achieved by the rotator. For example drifts in the orbit around the rotators have been correlated with drifts on the longitudinal orientation of the spin vector measured by the detectors.

TABLE I. Measured polarization lifetimes from FY12, FY13, FY15, and FY17 polarized proton runs with and without spin rotators. $*$ These are stores which used the same lattice as in FY12.

\begin{tabular}{lcc}
\hline \hline Lattice/Run & $\begin{array}{c}\text { Measured } \\
\text { Rot. Off [\%/hr] }\end{array}$ & $\begin{array}{c}\text { Measured } \\
\text { Rot. On [\%/hr] }\end{array}$ \\
\hline Blue p-p 255 GeV FY17 & $0.38 \pm 0.03$ & \\
Blue p-Au 100 GeV FY15 & $0.18 \pm 0.02$ & \\
Blue p-p 100 GeV FY15 & $0.95 \pm 0.02$ & $1.26 \pm 0.02$ \\
Blue p-p 255 GeV FY13* & & $0.839 \pm 0.05$ \\
Blue p-p 255 GeV FY12 & & $0.76 \pm 0.065$ \\
Yellow p-p 255 GeV FY17 & $0.42 \pm 0.03$ & \\
Yellow p-p 100 GeV FY15 & $0.5 \pm 0.02$ & $0.52 \pm 0.02$ \\
Yellow p-p 255 GeV FY13* & & $0.75 \pm 0.05$ \\
Yellow p-p 255 GeV FY12 & & $1.05 \pm 0.077$ \\
\hline \hline
\end{tabular}

TABLE II. Estimate of the strength of the effective imperfection spin resonance for RHIC Runs based Table I.

\begin{tabular}{lcc}
\hline \hline RHIC Lattice/Run & Rotator & $\begin{array}{c}\text { Estimate of } \\
\text { Effective Spin } \\
\text { Imperfection Range }\end{array}$ \\
\hline Blue p-p 255 GeV FY17 & Off & $0-0.05$ \\
Blue p-Au 100 GeV FY15 & Off & $0.05-0.1$ \\
Blue p-p 100 GeV FY15 & Off & $0.1-0.2$ \\
Blue p-p 100 GeV FY15 & On & 0.2 \\
Blue p-p 255 GeV FY13* & On & $0-0.05$ \\
Blue p-p 255 GeV FY12 & On & $0-0.05$ \\
Yellow p-p 255 GeV FY17 & Off & $0-0.05$ \\
Yellow p-p 100 GeV FY15 & Off & $0.1-0.2$ \\
Yellow p-p 100 GeV FY15 & On & $0.1-0.2$ \\
Yellow p-p 255 GeV FY13* & On & 0.05 \\
Yellow p-p 255 GeV FY12 & On & $0.05-0.1$ \\
\hline \hline
\end{tabular}

Using Table I and Eq. (38) with the approach described in the previous section, we can estimate an effective imperfection spin resonance for each of these runs. The effective imperfection represents the combined contribution from both the lattice and the rotators (if turned on). These estimates are summarized in Table II.

This estimate is accomplished by calculating the polarization lifetimes using the respective lattices for each run and including imperfection spin resonances from 0 to 0.2 . This corresponds to snake or rotator detuning of 0 to 36 degrees. These lifetimes are calculated for emittances and tunes which reflect the values using in operations, for example between $2.5-3.5 \mathrm{~mm}$-mrad normalized and fractional tunes between $0.683-0.691$.

\section{PROBLEM OF VERY LONG TRACKING}

While tracking over 1-2 hours delivers results consistent with observations, polarization loss seems overestimated by 1-2 percent per hour when the tracking is extended beyond 2 hours. In Fig. 11 we can see an example of a 6 hour tracking simulation. One of the interesting features is the existence of "kinks." At the moment it is unclear if this is a result of long-term numerical issues, or due to the fact that we are reaching the limits of this type of approximation. It is possible that the several simplifying assumptions made to the transverse, longitudinal, and spin motion make such long term estimates invalid. In addition beam diffusion and loss impact the larger emittance particles more than the small ones. Thus we are counting a significant number of particles which normally would have been lost and not weighed in the average polarization. In addition there are periodic kicks due to intrabeam scattering and beam-beam which could move the particles in and out of resonance tongue regions, so that particles do not reside in a higher order parametric resonance region long enough to see the strong effect observed in the simulation. 


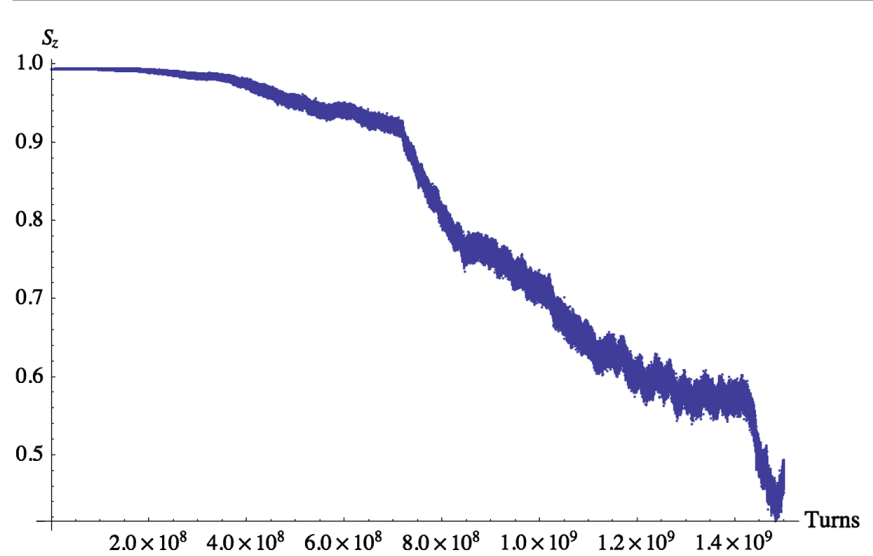

FIG. 11. 6 hour tracking for the FY17 $255 \mathrm{GeV}$ RHIC stored lattice.

\section{SUMMARY}

We have extended our lattice independent spin tracking code to include the effects of longitudinal dynamics. The contribution from longitudinal dynamics adds an oscillating betatron phase component to the complex first-order spin resonance strength. It also modulates the bare spin tune $G \gamma$ due to the contribution of the energy via $\delta$. In the single resonance model this added dynamics causes loss of polarization over which is otherwise absent in the single resonance model with orthogonal snakes.

Including multiple spin resonances without longitudinal dynamics also does not generate polarization loss on the timescale of several hours. However as in the single resonance case, the addition of longitudinal motion causes polarization losses on the order of $0.2 \%-1 \%$ /hour. This loss rate is consistent with observed loss rates in RHIC.

Our studies show that the existence of longitudinal dynamics is the most important factor in causing polarization loss for a stored beam. Although necessary to cause polarization loss, the actual magnitude of the longitudinal parameters possible in RHIC appears to have little significance. For example polarization loss was insensitive to the values of synchrotron tune, chromaticity, and longitudinal emittance.

After longitudinal dynamics, the next important factor in determining polarization loss for the stored beam, is the strength of the intrinsic and imperfection spin resonances, their numerology and proximity. Finally we have recast the single resonances T-BMT equation into a parametric resonance formalism including simplified longitudinal dynamics.

\section{ACKNOWLEDGMENTS}

I would like to thank Mike Blaskiewicz for his many fruitful discussions. Work supported by Brookhaven Science Associates, LLC under Contract No. DE-AC0298CH10886 with the U.S. Department of Energy. This research used resources of the National Energy Research Scientific Computing Center (NERSC), which is supported by the Office of Science of the U.S. Department of Energy under Contract No. DE-AC02-05CH11231.

[1] D. T. Abell, D. Meiser, V. H. Ranjbar, and D. P. Barber, Accurate and efficient spin integration for particle accelerators, Phys. Rev. Accel. Beams 18, 024001 (2015).

[2] F. Méot, The ray-tracing code Zgoubi-Status, Nucl. Instrum. Methods Phys. Res., Sect. A 767, 112 (2014).

[3] V. H. Ranjbar, F. Méot, M. Bai, D. T. Abell, and D. Meiser, Polarization response of RHIC electron lens lattices, Phys. Rev. Accel. Beams 19, 101002 (2016).

[4] V. H. Ranjbar, Approximations for crossing two nearby spin resonances, Phys. Rev. Accel. Beams 18, 014001 (2015).

[5] E. D. Courant and R. D. Ruth, The acceleration of polarized protons in circular accelerators, Brookhaven National Laboratory Technical Report No. BNL-51270, 1980.

[6] M. Bai, C. Allgower, L. Ahrens, J. Alessi, K. Brown, G. Bunce, P. Cameron, C. M. Chu, E. D. Courant, J. W. Glenn, H. Huang, D. Jeon, A. E. Kponou, K. Krueger, A. Luccio, Y. I. Makdisi, S. Y. Lee, L. Ratner, K. Reece, T. Roser et al., Observation of a Hybrid Spin Resonance, Phys. Rev. Lett. 84, 1184 (2000).

[7] S. Y. Lee, Accelerator physics (World Scientific Publishing, Singapore, 1999).

[8] E. D. Courant and R. D. Ruth, Spin motion and resonances in accelerators and storage rings, Brookhaven National Laboratory Technical Report No. C-A/AP 292 V2, 2008.

[9] G. H. Hoffstaetter, High-Energy Polarized Proton Beams: A Modern View, Springer Tracts in Modern Physics (Springer, New York, NY, 2006), Vol. 218.

[10] V. H. Ranjbar et al., Spin coupling resonance and suppression in the AGS, Phys. Rev. Accel. Beams 7, 051001 (2004).

[11] D. P. Barber, H. Mais, and G. Ripken, Conversion of the thin lens 8X8 matrix formalism of the spin orbit coupling (SLIM) to a formalism using dispersion, DESY Technical Report No. DESY-M-84-17, 1984.

[12] A. W. Chao and M. G. Minty, Polarization algorithm with SLIM: Solution by linear matrices, SLAC Technical Report No. SLAC-AP-105, 1996.

[13] M. Froissart and R. Stora, Depolarization of a beam of polarized protons in a synchrotron, Nucl. Instrum. Methods Phys. Res. 7, 297 (1960).

[14] S. Blanes, F. Casas, J. Oteo, and J. Ros, The Magnus expansion and some of its applications, Phys. Rep. 470, 151 (2009).

[15] S. Fartoukh and R. Jones, Determination of Chromaticity by the Measurement of Head-Tail Phase Shifts : Simulations, Results from the SPS and a Robustness Study for the LHC, CERN CERN-LHC-PROJECT-REPORT-602, 2002.

[16] C. Y. Tan and V.H. Ranjbar, Analytic approximation of the head-tail phase difference from continuous transverse excitation for measuring chromaticity, Phys. Rev. Accel. Beams 11, 032802 (2008).

[17] V. H. Ranjbar and P. Ivanov, Chromaticity and wakefield effect on the transverse motion of longitudinal bunch slices in the Fermilab Tevatron, Phys. Rev. Accel. Beams 11, 084401 (2008). 
[18] V. Ranjbar, A. Marusic, and M. Minty, in Proceedings, 5th International Beam Instrumentation Conference (IBIC 2016): Barcelona, Spain, 2016, edited by I. Costa, U. Iriso, F. Pérez, and V. R. W. Schaa (JACoW, Geneva, Switzerland, 2017), p. TUPG49.

[19] V. Lebedev, E. Lorman, V. H. Ranjbar, and A. Xiao, in Proceedings of the 9th European Particle Accelerator Conference, Lucerne, 2004 (EPS-AG, Lucerne, 2004) [http://accelconf.web.cern.ch/AccelConf/e04/].
[20] S. Y. Lee and M. Berglund, Overlapping spin synchrotron sideband resonances, Phys. Rev. E 54, 806 (1996).

[21] V.H. Ranjbar, T-bmt spin resonance tracker, https:// sourceforge.net/projects/t-bmt-spin-resonance-tracker/ (2018).

[22] R. polarimetery group, Final results for rhic polarized pp data analysis, https://wiki.bnl.gov/rhicspin/Results (2018).

[23] W. B. Schmidke et al., RHIC polarization for Runs 9-17, Brookhaven National Laboratory Technical Report No. BNL-209057-2018-TECH, 2018. 